\title{
Positivdruckverfahren bei Cheyne-Stokes-Atmung infolge chronischer Herzerkrankung
}

\author{
B. Schönhofer \\ T. Barchfeld \\ S. Suchi \\ J. Kerl \\ A. Simon \\ D. Köhler
}

Positive Pressure Ventilation in Cheyne-Stokes Respiration

\section{Zusammenfassung}

Die kongestive Kardiomyopathie mit Cheyne-Stokes-Atmung (CSA) ist mit einer hohen Mortalitätsrate assoziiert. Continuous positive airway pressure (CPAP) bessert die Prognose. Wir berichten in dieser retrospektiven Untersuchung über unsere Ergebnisse zur Differenzialtherapie mit unterschiedlichen Positivdruckverfahren bei CSA. Der Beobachtungszeitraum reichte von 1995-1999. Einschlusskriterien waren ein Respiratory Disturbance Index $>10 / \mathrm{h}$ und mehr als $50 \%$ zentrale und/oder gemischtförmige Apnoen. Jeder Patient wurde über jeweils 4 Wochen mit CPAP, Bilevel CPAP im Spontanmode (d.h. BiPAP S oder ST Modus) und BiPAP im kontrollierten Mode (d.h. BiPAP $\mathrm{T}$ ) behandelt. Responder für das jeweilige Positivdruckverfahren wurden nach subjektiven und objektiven Kriterien definiert. Es wurden 41 männliche Patienten eingeschlossen. Die Responder verteilten sich folgendermaßen: CPAP: $n=13$ Patienten $(31,7 \%)$, BiPAP S/ST: $\mathrm{n}=9$ Patienten $(22,0 \%)$ und BiPAP T: $\mathrm{n}=12$ Patienten $(29,3 \%)$. Insgesamt $7 / 41$ Patienten $(17,1 \%)$ lehnten eine langfristige Therapie mit einem Positivdruckverfahren ab. Die überwiegende Mehrheit der Patienten mit CSA lässt sich mit CPAP bzw. BiPAP S/ST befriedigend einstellen. Auch wenn sich in der BiPAP-T-Respondergruppe die Atmung im Schlaf weitgehend normalisierte, kam diese Beatmungsform langfristig nur bei etwa $29 \%$ der Patienten zur Anwendung.

\section{Abstract}

Cheyne-Stokes respiration (CSR) is found in patients with chronic left ventricular failure and associated with a reduced prognosis. Continuous positive airway pressure (CPAP) improves the survival rate. In this retrospective study we report on the effect of different positive pressure ventilation modes in CSR. The observation period lasted from 1995 - 1999. Inclusion criteria was CSR with a respiratory disturbance index $>10 /$ h whereas $>50 \%$ of the events had to be central and/or mixed. In each patient a 4 week lasting intervention with each of CPAP, Bilevel CPAP in spontan mode (i.e. BiPAP S or BiPAP ST) and BiPAP in controlled mode (i.e. BiPAP T) was performed. Responder were defined by subjective and objective criteria. In total 41 males were included. Responder were distributed as follows: CPAP: $n=13$ patients (31.7\%), BiPAP S/ST: $\mathrm{n}=9$ patients $(22.0 \%)$ and BiPAP T: $\mathrm{n}=12$ patients $(29.3 \%)$. In total 7 from 41 patients $(17.1 \%)$ rejected a long-term treatment with positive pressure ventilation.

The majority of patients with CSR responded to CPAP and BiPAP $\mathrm{S} / \mathrm{ST}$ mode. Compared to the other responder groups the best quality was reached with BiPAP T. However the latter was performed only by $29 \%$ of the population.

\section{Einleitung}

Unterschiedliche Erkrankungsgruppen führen $\mathrm{zu}$ zentralen schlafbezogenen Atmungsstörungen. Neben zentralnervösen Erkrankungen (wie z.B. Enzephalitis oder Hirninsult) die primär zur Irritation des Atmungszentrums führen, treten zentrale schlafbezogene Atmungsstörungen idiopathisch oder sekundär vor allem bei kongestiver Kardiomyopathie in Form der CheyneStokes-Atmung (CSA) auf [1]. CSA ist gekennzeichnet durch periodisch auftretende zentral bedingte Apnoen und/oder Hypo-

Krankenhaus Kloster Grafschaft, Zentrum für Pneumologie, Beatmungs- und Schlafmedizin, Schmallenberg-Grafschaft (Prof. Dr. Dieter Köhler) 
pnoen gefolgt von Hyperventilation in einem Crescendo-Decrescendo-artigen Muster.

Die bei Herzerkrankung mit einer Mortalität von etwa $40 \%$ einhergehende CSA [2-4] resultiert aus einer komplexen kardiorespiratorischen Interaktion, deren Pathophysiologie zur Zeit noch nicht eindeutig aufgeklärt ist $[1,5]$.

In der Therapie der CSA kommen neben der Pharmakotherapie der chronischen Herzinsuffizienz (z.B. in Form von ACE-Hemmern, Diuretika; Theophyllin und eventuelle Betablocker $[6,7])$, die Sauerstofflangzeittherapie $[8,9]$ und Positivdruckbeatmung zur Anwendung. Neben continuous positive airway pressure (CPAP) $[10,11]$ wurden in jüngster Vergangenheit Bilevel-CPAPGeräte und adaptive pressure support Servo-Ventilation eingesetzt $[12,13]$.

In diesem Artikel berichten wir über die Ergebnisse einer retrospektiven Studie, in der Patienten mit CSA sequenziell mit drei unterschiedlichen Positivdruckverfahren behandelt wurden.

\section{Patienten und Methodik}

\section{Ein- und Ausschlusskriterien}

Innerhalb des Beobachtungszeitraums von 1995-1999 wurden Patienten mit CSA bei kongestivem Verlauf einer chronischen Herzinsuffizienz in diese Studie eingeschlossen, wenn sich in der Polygraphie (bzw. Polysomnographie) das typische Muster der CSA ergab. Darüber hinaus mussten der Respiratory Disturbance Index (RDI) größer 10/h und mehr als 50\% der Apnoen zentral und/oder gemischtförmig sein. Chronische Herzinsuffizienz war definiert über eine echokardiographisch nachgewiesene Ejektionsfraktion von $<40 \%$ bei New York Heart Association (NYHA) Klassifikation Stadium II und III. Die Patienten mit chronischer Herzinsuffizienz mussten seit $>4$ Wochen klinisch stabil und medikamentös mit z.B. Diuretika, ACE-Hemmer, Betablocker und Digoxin therapiert sein. Patienten mit komplexen Arrhythmien, relevanten Herzklappenfehlern und mit dominierender obstruktiver Schlafapnoe (> 50\% obstruktive Ereignisse) wurden ausgeschlossen.

\section{Studienprotokoll}

Die Einstellung der Patienten auf die jeweiligen Verfahren erfolgte unter kontinuierlicher klinischer und polygraphischer Überwachung durch technisches und ärztliches Personal im Schlaflabor. Initial wurde immer mit CPAP (Max bzw. Max II, Firma MAP; Martinsried und Sullivan IV bzw. Ivc, Firma ResMed, Mönchengladbach) behandelt. In Abhängigkeit von subjektiver Befindlichkeit und polygraphisch erhobenem Befund wurde der CPAP-Druck während einer 2- bis 3-tägigen Einstellungsphase erhöht. Bei Patienten mit zugrunde liegender Herzerkrankung wurde mit einem CPAP von $5 \mathrm{~cm} \mathrm{H}_{2} \mathrm{O}$ begonnen. Der maximale CPAP-Druck war $12 \mathrm{~cm} \mathrm{H}_{2} \mathrm{O}$, umso eine potenzielle Beeinträchtigung der Hämodynamik zu verhindern. Die Patienten wurden nach der jeweiligen Einstellungsphase für 4 Wochen nach Hause entlassen. Bei der Verlaufskontrolle wurde der Patient nach seiner subjektiven Einschätzung der CPAP-Therapiequalität befragt. Die in die Analyse der Studie eingehenden polygraphischen Messergebnisse wurden nach Ablauf der Erprobungsphase des jeweiligen Gerätes mit identischer Druckeinstellung erhoben.
Ein Apnoe-Index (AI) < 50\% des Ausgangsbefundes unter CPAP wurde als objektiver Therapieerfolg gewertet. Bei gleichzeitig bestehender subjektiver Besserung wurde auf weitere Therapieversuche mit alternativen Positivdruckverfahren verzichtet.

Im Falle eines $\mathrm{AI}>50 \%$ des Ausgangsbefundes wurde dem Patienten angeboten, die nächste Therapiephase mit einem technisch höherwertigen Gerät zu beginnen. Wurde dieses Vorgehen jedoch bei subjektiv empfundener Effektivität trotz fehlendem objektiven Therapieerfolg vom Patienten nicht gewünscht, wurde CPAP verordnet. War der Patient mit der Therapieumstellung einverstanden, wurde auf Bilevel CPAP, d. h. „BiPAP“ im Spontanmode (BiPAP S oder BiPAP ST mit vorgegebener Atemfrequenz [so genannte „backup-Frequenz"] bis maximal 12/min umgestellt. Die Druckfindung von IPAP (Inspiratory positive airway pressure) und EPAP (Exspiratory positive airway pressure) orientierte sich am zuvor angewandten CPAP-Druck (d.h. die Differenz zwischen IPAP und EPAP war $>4 \mathrm{~cm} \mathrm{H}_{2} \mathrm{O}$ und der Mittelwert aus beiden Werten orientierte sich am CPAP). Auch dieser Einstellungsphase folgte eine 4-wöchige Erprobungsphase unter häuslichen Bedingungen. Bei der sich anschließenden Verlaufskontrolle unter stationären Bedingungen wurde bezüglich subjektiver und objektiver Beurteilung der Einstellungsqualität und der Verordnung des BiPAP S/ST-Gerätes wie oben unter CPAP beschrieben vorgegangen. War der Patient bei ebenfalls unbefriedigendem Therapieergebnis mit einem weiteren Therapieversuch einverstanden, schloss sich zuletzt die Einstellung auf BiPAP im kontrollierten Modus (d.h. BiPAP T) mit anschließender 4-wöchiger Erprobungsphase. Bei der Einstellung auf diesen Beatmungsmodus wurden IPAP und EPAP aus der vorhergehenden Beatmungsphase berücksichtigt. Allerdings bestand die Option, beide Druckniveaus dem subjektiven Wohlbefinden der Patienten anzupassen.

Vor individueller Einstellung des Beatmungsverfahrens in den BiPAP-T-Modus wurde das spontane Atemmuster der Patienten (d.h. Atemfrequenz, Inspirations- und Exspirationszeit) im Wachzustand mittels CP100 Bicore (Firma Medilab, Estenfeld) ermittelt. Die Atemfrequenz des Beatmungsgerätes wurde geringgradig oberhalb der Spontanatmungsfrequenz eingestellt.

Letztlich bestand für die Patienten mit CPAP- und/oder BiPAPS/ST-Inakzeptanz auch die Möglichkeit, bereits während der initialen Adaptationsphase auf das jeweils höherwertige Beatmungsverfahren (d.h. BiPAP S/ST oder BiPAP T) eingestellt zu werden. Beim BiPAP-S/ST und -T-Modus wurden ausschließlich BiPAP-Geräte der Firma Respironics verwandt.

Nach Abschluss der maximal 3-monatigen Beobachtungsphase wurden die Responder für das jeweilige Positivdruckverfahren anhand der definitiven Geräteverordnung festgestellt, die sich neben einem objektiven Kriterium im wesentlich an dem vom Patienten bevorzugten Verfahren orientierte.

Bei fehlender Akzeptanz bestand für die Patienten die Option, die Therapie jederzeit definitiv zu beenden und eine nächtliche Sauerstofftherapie durchzuführen.

\section{Messungen}

Die polygraphischen Parameter wurden mit Poly-MESAM (MAP, Martinsried, Deutschland) ermittelt. Eine Sonderanfertigung er- 
möglichte es, die polygraphische Aufzeichnung von 16 Plätzen online auf die Bildschirmeinheiten in den Überwachungsraum zu übertragen. Die Atmung bzw. Atmungsmechanik wurden mit einem oronasal applizierten Thermistor, einem Thorax- und Bauchgurt erfasst. Die Sauerstoffsättigung $\left(\mathrm{SaO}_{2}\right)$ wurde mit Pulsoximetrie gemessen. Der Diagnose CSA lag der Nachweis des typisch periodischen Atemmusters zugrunde [3]. Eine Apnoe wurde nach komplettem Sistieren des Thermistorsignals über mindestens 10 Sekunden registriert. Bei obstruktiven Apnoen wurde neben sistierendem Flow weiterhin Bewegung der Bauchund Thoraxgurte nachgewiesen. Zentrale Apnoen wurden gewertet, wenn neben dem mindestens 10 Sekunden andauernden Sistieren des Flowsignals gleichzeitig keine Signale über Bauch bzw. Thorax registriert wurden. Gemischtförmige Apnoen wurden gewertet, wenn sich einem initial zentralen Ereignis ein obstruktiver Anteil anschloss [14]. Die Hypopnoe wurde über eine Reduktion des Flows $>50 \%$ mit einer Zeitdauer von mehr als 10 s definiert.

Eine systolische Funktionsstörung wurde mittels Echokardiographie bei einer Ejektionsfraktion von kleiner $40 \%$ angenommen [15].

Zur Erfassung der Tagesschläfrigkeit wurde die Epworth-Schläfrigkeitsskala (ESS) verwandt [16].

\section{Statistik}

Alle Werte sind als Mittelwert \pm Standardabweichung (SD) angegeben.

Für jeden Zielparameter (RDI, AI gesamt, AI zentral, gemischt und obstruktiv, $\mathrm{SaO}_{2}$ Mittel und Nadir und ESS) wurde eine zweifaktorielle Varianzanalyse (ANOVA) mit Messwiederholung durchgeführt. Faktor 1 war die Respondergruppe (betweengroups), Faktor 2 die Messwiederholung vor und nach der Intervention (within-subjects).

Alle Analysen wurden mit dem Programm „Statistica“, Version 5.5 durchgeführt [17]. Ein zweiseitiges $p<0,05$ wurde als signifikant bewertet.

\section{Ergebnisse}

Im 5-jährigen Beobachtungszeitraum behandelten wir insgesamt 41 Patienten (ausschließlich Männer, Alter: 62,5 \pm 7,6 Jahre, BMI: $31,6 \pm 5,1 \mathrm{~kg} / \mathrm{m}^{2}$ mit CSA). Der mittlere RDI war $46,7 \pm$ 23,8/h, der mittlere AI war 26,4 $\pm 21,0 / \mathrm{h}$. Zehn Patienten zeigten Vorhofflimmern. Die Blutgase vor Therapiebeginn (ohne Sauerstoffinsufflation) waren in der Gesamtpopulation: PO2: 70,6 \pm 8,8, PCO2: $38,4 \pm 3,6 \mathrm{~mm} \mathrm{Hg}$, pH: 7,41 $\pm 0,04$, HCO3: $23,2 \pm$ $1,3 \mathrm{mmol} / \mathrm{l}$ und $\mathrm{BE}: 2,6 \pm 5,2$.

Bei 41 Patienten wurde ein Therapieversuch mit den 3 verschiedenen Positivdruckverfahren in gleicher Reihenfolge unternommen. Dreizehn Patienten wünschten nach subjektiv erfolgreich eingeschätzter CPAP-Therapie keinen weiteren Therapieversuch (Tab.1) und wurden daher nach der Probephase definitiv mit CPAP versorgt. Bei 2 Patienten aus diesem Kollektiv wurde der objektive Therapieerfolg jedoch nicht erreicht. Analog verhielt es sich mit 9 Patienten (Tab.1), bei denen nach jeweils 4-wöchi-
Tab. 1 Anzahl der Patienten in den verschiedenen Therapie-Phasen.

\begin{tabular}{llll}
\hline & $\begin{array}{l}\text { CPAP } \\
(\mathbf{n}=41)\end{array}$ & $\begin{array}{l}\text { BiPAP S/ST } \\
(\mathbf{n}=25)\end{array}$ & $\begin{array}{l}\text { BiPAP T } \\
(\mathbf{n}=16)\end{array}$ \\
\hline $\begin{array}{l}\text { subjektiv gut - objektiv gut } \\
\text { (Al }<50 \%)\end{array}$ & $11^{*}$ & $7 \#$ & 12 \\
$\begin{array}{l}\text { subjektiv gut - objektiv schlecht } \\
\text { (Al }>50 \%)\end{array}$ & $2^{*}$ & $2 \#$ & 0 \\
\hline \begin{tabular}{l} 
Therapieversuch abgebrochen \\
\hline
\end{tabular} & 3 & 0 & 4 \\
\hline
\end{tabular}

* Patienten, die kein BiPAP S/ST oder BiPAP T wünschen.

\# Patienten, die kein BiPAP T wünschen. Apnoe-Index (Al)

ger CPAP und folgender BiPAP S/ST-Phase auf eigenen Wunsch letzteres Verfahren verordnet wurde, obwohl bei 2 Patienten aus diesem Kollektiv der objektive Therapieerfolg nicht erreicht wurde.

Insgesamt 7/41 Patienten (17,1\%) lehnten eine langfristige Therapie mit einer Positivdruckbeatmung ab. Drei von diesen Patienten brachen die Therapie frühzeitig definitiv ab und wurden mit Sauerstoff versorgt (7,3\%). Insgesamt 4 Patienten $(9,8 \%)$ durchliefen zwar alle 3 Therapieverfahren, akzeptierten aber langfristig keine Intervention.

Die Responder verteilten sich folgendermaßen auf die Beatmungsverfahren: CPAP: $\mathrm{n}=13$ Patienten (31,7\%), BiPAP S/ST: $\mathrm{n}=9$ Patienten $(22,0 \%)$ und BiPAP T: $\mathrm{n}=12$ Patienten $(29,3 \%)$. Die Beatmungsdrucke (Mittelwert und SD) der jeweiligen Verfahren sind in Abb. 1 dargestellt.

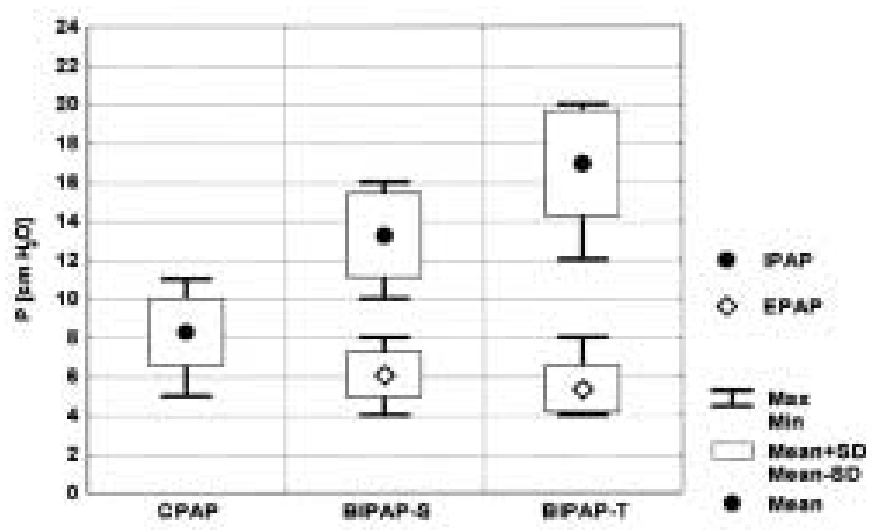

Abb. 1 Druckwerte (maximal, minimal, Mittel \pm Standardabweichung) von CPAP, BiPAP S/ST und BiPAP T (hier IPAP und EPAP).

Die RDI-, AI- und $\mathrm{SaO}_{2}$-Mittelwerte der Respondergruppen vor Beginn und mit der Intervention sind in Tab. 2 aufgeführt. Es ergab sich eine generelle Absenkung von RDI und AI bzw. Anhebung der Sauerstoffsättigung unter der Therapie $(p<0,02$ in allen Fällen) (Abb. 2-4). Es gab keine generellen Unterschiede zwischen den Respondergruppen ( $p>0,3$ in allen Fällen). Die Wirksamkeit der Verfahren, bezogen auf die jeweilige Respondergruppe, unterschied sich nicht ( $p>0,18$ in allen Fällen). Jedes Verfahren führte zu einer signifikanten Reduktion der ESS (Tab. 2). 
Tab. 2 Respiratory Disturbance Index (RDI), Al (zentral, gemischt und obstruktiv), Sauerstoffsättigung (SaO ${ }_{2}$ ) und Epworth Sleepiness Skala (ESS) zur Baseline und unter der Intervention mit CPAP, BiPAP S/ST und BiPAP T in der jeweiligen Respondergruppe

\begin{tabular}{|c|c|c|c|c|c|c|c|}
\hline \multirow[t]{2}{*}{ Responder } & \multicolumn{2}{|c|}{$C P A P(n=13)$} & \multicolumn{2}{|c|}{ BiPAP S/ST (n = 9) } & \multicolumn{2}{|c|}{ BiPAP T $(n=12)$} & \multirow{2}{*}{$\begin{array}{l}\text { p-Wert } \\
\text { Intervention }\end{array}$} \\
\hline & Baseline & Intervention & Baseline & Intervention & Baseline & Intervention & \\
\hline RDI (/h) & $49,1 \pm 17,6$ & $26,8 \pm 16,8$ & $44,6 \pm 30,7$ & $22,6 \pm 12,7$ & $49,7 \pm 16,6$ & $14,1 \pm 13,6$ & 0,0001 \\
\hline$A l(/ h)$ & $33,5 \pm 24,4$ & $9,6 \pm 9,9$ & $21,3 \pm 18,4$ & $7,7 \pm 12,1$ & $34,6 \pm 17,5$ & $1,4 \pm 2,0$ & 0,0001 \\
\hline Al - zentral (/h) & $5,5 \pm 6,4$ & $2,2 \pm 5,4$ & $6,8 \pm 8,6$ & $1,9 \pm 2,3$ & $9,3 \pm 10,9$ & $0,4 \pm 1,1$ & 0,0010 \\
\hline$A l$ - gemischt $(/ h)$ & $14,3 \pm 16,0$ & $2,0 \pm 3,6$ & $7,0 \pm 8,3$ & $2,1 \pm 2,7$ & $12,1 \pm 12,1$ & $0,0 \pm 0,0$ & 0,0012 \\
\hline Al - obstruktiv (/h) & $13,6 \pm 15,6$ & $5,4 \pm 6,0$ & $7,4 \pm 9,5$ & $3,7 \pm 8,1$ & $12,2 \pm 10,6$ & $0,5 \pm 0,8$ & 0,0033 \\
\hline $\mathrm{SaO}_{2}-$ Mittel (\%) & $91,6 \pm 4,2$ & $94,7 \pm 1,8$ & $91,5 \pm 1,7$ & $93,0 \pm 2,1$ & $92,1 \pm 2,1$ & $93,5 \pm 1,7$ & 0,0008 \\
\hline $\mathrm{SaO}_{2}-\mathrm{Nadir}(\%)$ & $68,1 \pm 15,1$ & $80,2 \pm 13,7$ & $71,1 \pm 14,4$ & $77,9 \pm 8,8$ & $74,1 \pm 8,2$ & $81,5 \pm 4,3$ & 0,0008 \\
\hline ESS & $10,3 \pm 4,1$ & $6,5 \pm 3,8$ & $11,8 \pm 2,5$ & $8,3 \pm 2,3$ & $12,4 \pm 4,5$ & $9,2 \pm 4,1$ & 0,0001 \\
\hline
\end{tabular}

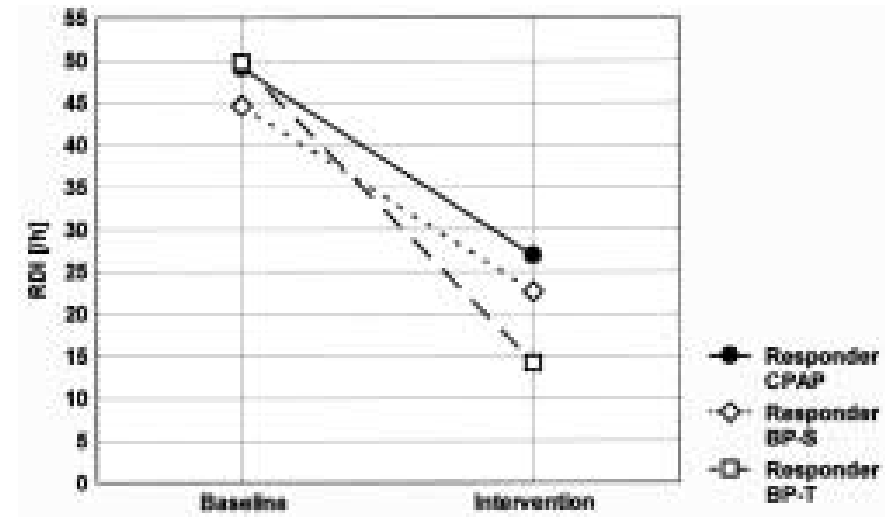

Abb. 2 RDI vor und unter der Intervention mit CPAP, BiPAP S/ST und BiPAP T in der jeweiligen Respondergruppe.

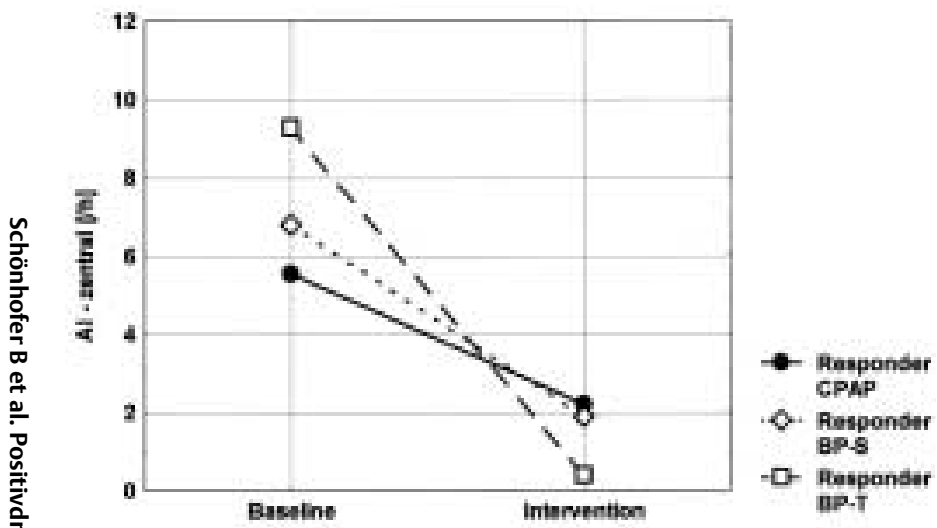

Abb. 3 Al (zentral) vor und unter der Intervention mit CPAP, BiPAP S/ST und BiPAP T in der jeweiligen Respondergruppe.

Durch BiPAP im S- bzw. ST-Modus kam es im Vergleich zu CPAP zu keiner nennenswerten Steigerung des Therapieeffektes. In der BiPAP-T-Respondergruppe ergab sich im Vergleich zu CPAP unter Anwendung von BiPAP S/ST sogar ein schlechteres Ergebnis.

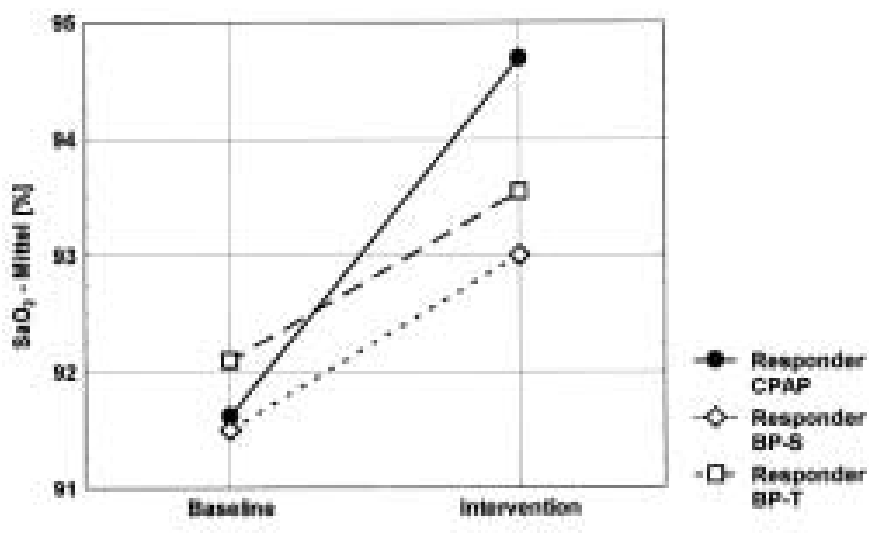

Abb. 4 Mittlerer $\mathrm{SaO}_{2}$ vor und unter der Intervention mit CPAP, BiPAP $\mathrm{S} / \mathrm{ST}$ und BiPAP T in der jeweiligen Respondergruppe.

\section{Diskussion}

Die wesentlichen Ergebnisse unserer Studie sind folgende:

Bei CSA ist ein therapeutischer Stufenplan bestehend aus verschiedenen Positivdruckverfahren sinnvoll. In der jeweiligen Respondergruppe kam es zur signifikanten Besserung der Atmung im Schlaf. Es ließ sich kein genereller Unterschied in der Wirksamkeit der einzelnen Verfahren nachweisen.

Bei CSA gelingt es häufig nicht, mit CPAP eine befriedigende Therapieeinstellung zu erreichen. Im Einzelfall können dann durch BiPAP im S- bzw. ST-Modus bessere Einstellungsergebnisse erzielt werden. Dies wurde kürzlich auch für variable positive airway pressure (VPAP) II ST (Firma Resmed, Australien) gezeigt [13].

Im Gesamtkollektiv führten jedoch BiPAP im S- bzw. ST-Modus im Vergleich zu CPAP zu keiner nennenswerten Steigerung des Therapieeffektes. Nur bei etwa einem Drittel der Patienten erfolgte langfristig die Beatmung im kontrollierten Beatmungsmodus mit Druckvorgabe (BiPAPT), obwohl sich hierunter die Atmung im Schlaf weitgehend normalisierte. Nur eine Minderheit (etwa 17\%) der Gesamtpopulation lehnte eine langfristige Positivdruckbeatmung ab. 
Vor der Diskussion der Ergebnisse werden zunächst die relevanten Limitationen der Studie abgehandelt:

1. Das Studiendesign war nicht kontrolliert-randomisiert. Hierdurch entfällt ein wesentliches Qualitätskriterium der evidenzbasierten Medizin. Insbesondere ist anzunehmen, dass die vorgegebene Reihenfolge der Geräte in unserer Studie die Geräteauswahl beeinflusst hat bzw. die randomisierte Zuordnung einen relevanten Einfluss auf die subjektive Einschätzung der Patienten gehabt hätte.

2. Aufgrund der alltäglichen Belastung im Schlaflabor standen bei der Auswertung der Ergebnisse nur polygraphisch erhobene Daten komplett zur Verfügung. Auch wenn polysomnographisch erhobene Daten bei einer Reihe von Patienten vorhanden sind, wurde in diesem Artikel mangels konsistenter Datenerhebung auf die Präsentation von Ergebnissen bez. Schlafarchitektur und Arousals verzichtet. Möglicherweise hätten sich aus polygraphischen Befunden weiter Erkenntnisse zur Einschätzung der Verfahren durch die Patienten ergeben.

3. Eine differenzierte Evaluation der subjektiven Einschätzung der unterschiedlichen Therapieverfahren zu Akzeptanz und Effekt der unterschiedlichen Therapieformen durch die Patienten liegt nicht vor. Es ist anzunehmen, dass eine solche Erhebung weitere Erkenntnisse $\mathrm{zu}$ diesen Aspekten ergeben hätte.

Trotz dieser Limitationen liefert diese Studie einige neue Erkenntnisse, die mitteilungswürdig sind, da unseres Wissens bisher keine vergleichbaren Untersuchungen zur Differenzialtherapie mit unterschiedlichen Positivdruckverfahren bei CSA mit längerem Beobachtungszeitraum vorliegen.

Die rein zentralen Apnoen liegen in den einzelnen Gruppen dieser Untersuchung lediglich zwischen etwa $20 \%$ und $30 \%$. Es ist aber wesentlich, dass bei allen Patienten die CSA im Vordergrund stand. Analog hierzu überwogen bei allen Patienten die zentralen in Kombination mit gemischtförmigen Apnoen die rein obstruktiven Apnoen. Es war jedoch unter Berücksichtigung der Periodizität der CSA manchmal sehr schwierig, die realen Ereignisse den klassischen Definitionen der Apnoe-Typen zuzuordnen.

Basierend auf den Ergebnissen dieser Studie kann es sinnvoll sein, beim Patienten mit CSA einen therapeutischen Stufenplan z. B. bestehend aus CPAP, BiPAP S/ST und BiPAP T zu durchlaufen. Dieses Prozedere ermöglicht es im Zweifelsfall, in Abhängigkeit von subjektiver Einschätzung durch den Patienten (bez. Therapieeffekt und Toleranz) und objektivem Messergebnis das individuell optimale Therapieverfahren zu ermitteln.

Für den klinischen Alltag ist die in dieser Studie gewählte Vorgehensweise häufig zu zeitintensiv. Sie bietet sich aber als mögliche therapeutische Option für Patienten mit CSA, die mit CPAP unzureichend therapiert ist.

Die Responderrate bez. der einzelnen Therapieverfahren war weitgehend homogen verteilt. Innerhalb jeder Respondergruppe kam es zu einer signifikanten Besserung der Atmung, ohne dass sich die Gruppen in der Wirksamkeit der einzelnen Verfahren signifikant unterschieden.
Auch wenn die kontrollierte Beatmung zum optimalen Ergebnis bezüglich der Atmung im Schlaf führte, kam dieses Verfahren längerfristig nur von der Minderheit unseres Kollektivs zum Einsatz. Es kann über die zugrunde liegenden Ursachen hierfür nur spekuliert werden. Möglicherweise führte aber die Beeinträchtigung der Eigenatmung durch das vorgegebene Beatmungsmuster bei diesem Modus zur Irritation der Patienten.

Der Nachweis von CSA geht bei Patienten mit chronischer Herzerkrankung mit einer erhöhten Mortalität einher [2,18]. Darüber hinaus führen ein Apnoe/Hypopnoe-Index > 30/Stunde, eine abgeschwächte Empfindlichkeit des Baroreflexes und eine reduzierte Herzfrequenzvariabilität zur Verschlechterung der Prognose der Patienten mit CSA [18]. Vor allem unter hämodynamischem Gesichtspunkt ist es bei herzkranken Patienten mit CSA sinnvoll, eine Positivdruckbehandlung durchzuführen. Die Arbeitsgruppe um Bradley konnte in mehreren physiologisch konzipierten Arbeiten zeigen, dass es mit Hilfe von CPAP auch ohne gleichzeitige Normalisierung der Atmung zur Besserung der Hämodynamik und Reduktion des Sympathikotonus kommt $[19,20]$. Auch wenn sich die Einstellungskriterien unter Schlaflaborbedingungen in dieser Studie auf die Besserung der Atmung im Schlaf konzentrierten, werden Sympathikotonus und Hämodynamik wahrscheinlich ebenfalls günstig beeinflusst.

In jüngerer Vergangenheit ließ sich auch im randomisierten und kontrollierten Studiendesign nachweisen, dass CPAP bei Patienten mit CSA und Ejektionsfraktion $<35 \%$ zur Verbesserung der Prognose führt [11]. Die zugrunde liegenden Wirkmechanismen bleiben zur Zeit allerdings offen. Neben der interventionellen Therapie mit Positivdruckverfahren ist jedoch eine Optimierung der kardialen Therapie unverzichtbar. So wurde kürzlich gezeigt, dass eine intensive medikamentöse Therapie mit ACE-Hemmern, Diuretika, Nitraten und $\beta$-Blockern zur Reduktion des pulmonary capillary wedge pressure und damit verbunden der schlafbezogenen Atmungsstörung führen [21].

Bez. der hämodynamischen Effekte verschiedener Formen der Positivdruckbeatmung bei chronischer Herzinsuffizienz existieren unterschiedliche Studienergebnisse [22-26]. In Abhängigkeit vom Beatmungsmodus bzw. -einstellung und der Charakteristik des untersuchten Patientengutes kam es unter Positivdruckbeatmung zu Besserung [22,23], aber auch zur Verschlechterung der Hämodynamik [24-26]. Bei zu hohem Druck und schlechter linksventrikulärer Funktion kann es unter Positivdruckbeatmung zur Kreislaufdepression kommen. In unserer Studie wurden keine hämodynamischen Messungen durchgeführt. Es kam während des Beobachtungszeitraums jedoch bei keinem Patienten zu einer klinisch relevanten Nebenwirkung. Ein wesentlicher Grund hierfür war sicherlich, dass nur klinisch stabile Patienten mit Herzinsuffizienz vom Typ NYHA II-III, die medikamentös regelrecht behandelt waren, eingeschlossen wurden. Darüber hinaus wurden die diensttuenden Ärzte und das technische Personal immer mit klar definierten, defensiven Titrationsvorgaben für die Adaptationsnächte versorgt.

Wir verzichten im Ergebnisteil auf die weitere Aufschlüsselung der zugrunde liegenden Herzerkrankungen (z.B. in primär dilatative Kardiomyopathie bzw. koronare Herzerkrankung), da vor bzw. während des Beobachtungszeitraums nicht bei allen Patienten eine Linksherzkatheteruntersuchung durchgeführt wur- 
de. Auch verfügten wir nur in der Minderheit der Patienten über Röntgenthoraxbilder, weshalb wir auf deren weitere Analyse im Rahmen dieser Untersuchung verzichteten. Anhand des bei uns erhobenen Echokardiographiebefundes lag jedoch bei allen Patienten mit Herzerkrankung und CSA eine relevante Störung der systolischen Pumpfunktion des linken Ventrikels vor.

Seit kurzem steht zur Behandlung der CSA bei Patienten mit Herzerkrankung ein neuer Therapiealgorithmus in Form des AutoSet CS-Gerätes (Firma Resmed, Mönchengladbach) zur Verfügung. Die kürzlich veröffentlichten Ergebnisse zu diesem neuen Therapieverfahren sind vielversprechend [12]. Da AutoSet CS im Beobachtungszeitraum dieser Studie nicht verfügbar war, konnte dieses Verfahren hier nicht berücksichtigt werden. Es muss jedoch in zukünftigen Studien untersucht werden, welchen Stellenwert AutoSet CS in der Differenzialtherapie des ZSA insbesondere im Vergleich zu BiPAP T hat.

Es ist sinnvoll, bei CSA einen therapeutischen Stufenplan mit unterschiedlichen Positivdruckverfahren zu durchlaufen, um die jeweiligen Responder in Abhängigkeit von subjektiver und objektiver Befundkonstellation zu eruieren.

\section{Literatur}

${ }^{1}$ Andreas S. Central sleep apnea and chronic heart failure. Sleep 2000; 23 Suppl 4: 220-223

${ }^{2}$ Hanly PJ, Zuberi-Khokhar NS. Increased mortality associated with Cheyne-Stokes respiration in patients with congestive heart failure. Am J Respir Crit Care Med 1996; 153: 272 - 276

${ }^{3}$ Javaheri S, Parker TJ, Wexler L et al. Occult sleep-disordered breathing in stable congestive heart failure. Ann Intern Med 1995; 122: 487-492

${ }^{4}$ Javaheri S, Parker TJ, Liming JD et al. Sleep apnea in 81 ambulatory male patients with stable heart failure. Circulation 1998; 97: 2154-2159

${ }^{5}$ Bradley TD, Floras JS. Pathophysiologic and therapeutic implications of sleep apnea in congestive heart failure. J Card Fail 1996; 2: 223 - 240

${ }^{6}$ Armstrong PW, Moe GW. Medical advances in the treatment of congestive heart failure. Circulation 1993; 88: 2941 - 2952

7 Javaheri S, Parker TJ, Wexler L et al. Effect of theophylline on sleep disordered breathing in heart failure. N Engl J Med 1996; 335: 562 - 567
${ }^{8}$ Franklin KA, Eriksson P, Sahlin C et al. Reversal of central sleep apnea with oxygen. Chest 1997; 111: $163-169$

${ }^{9}$ Javaheri S, Ahmed M, Parker TJ et al. Effects of nasal O2 on sleep-related disordered breathing in ambulatory patients with stable heart failure. Sleep 1999; 22: 1101 - 1106

${ }^{10}$ Naughton MT, Liu PP, Bernard DC et al. Treatment of congestive heart failure and Cheyne-Stokes respiration during sleep by continuous positive airway pressure. Am J Respir Crit Care Med 1995; 151: 92 - 97

11 Sin DD, Logan AG, Fitzgerald FS et al. Effects of continuous positive airway pressure on cardiovascular outcomes in heart failure patients with and without Cheyne-Stokes respiration. Circulation 2000; 102: $61-66$

12 Teschler H, Döhring J, Wang YM et al. Adaptive pressure support servo-ventilation. Am J Respir Crit Care Med 2001; 164: 614-619

${ }^{13}$ Willson GN, Wilcox P, Piper AJ et al. Noninvasive pressure preset ventilation for the treatment of Cheyne-Stokes respiration during sleep. Eur Respir J 2001; 17: 1250 - 1257

14 Tobin MJ, Cohn MA, Sackner MA. Breathing abnormalities during sleep. Arch Intern Med 1983; 143: 1221 - 1228

${ }^{15}$ Feigenbaum H. Echocardiography. Philadelphia: Lea \& Febiger, 1994

${ }^{16}$ Johns MW. Sleepiness in different situations measured by the Epworth Sleepiness Scale. Sleep 1994; 17: 703 - 710

17 StatSoft. STATISTICA for Windows. In: Tulsa, OK 2000

${ }^{18}$ Lanfranchi PA, Braghiroli A, Bosimini E et al. Prognostic value of nocturnal Cheyne-Stokes Respiration in chronic heart failure. Circulation 1999; (99): $1435-1440$

${ }^{19}$ Bradley TD. Hemodynamic and sympathoinhibitory effects of nasal CPAP in congestive heart failure. Sleep 1996; 19 (10 Suppl): 232 - 235

${ }^{20}$ Kaye DM, Mansfield D, Aggarwal A et al. Acute effects of continuous positive airway pressure on cardiac sympathetic tone in congestive heart failure. Circulation 2001; 103: 2336-2338

${ }^{21}$ Solin P, Bergin P, Richardson M et al. Influences of pulmonary capillary wedge pressure on central apnea in heart failure. Circulation 1999; 99: $1574-1579$

22 Acosta B, DiBenedetto R, Rahimi A et al. Hemodynamic effects of noninvasive bilevel positive airway pressure on patients with chronic congestive heart failure with systolic dysfunction. Chest 2000; 118: $1004-1009$

${ }^{23}$ Bradley TD, Holloway RM, McLaughlin PR et al. Cardiac output response to continuous positive airway pressure in congestive heart failure. Am Rev Respir Dis 1992; 145: 377-382

${ }^{24}$ Liston R, Deegan PC, McCreery C et al. Haemodynamic effects of nasal continuous positive airway pressure in severe congestive heart failure. Eur Respir J 1995; 8: 430-435

${ }^{25}$ Kiely JL, Deegan P, Buckley A et al. Efficacy of nasal continuous positive airway pressure therapy in chronic heart failure: importance of underlying cardiac rhythm. Thorax 1998; 53: 957-962

26 Philip-Joet FF, Paganelli FF, Dutau HL et al. Hemodynamic effects of bilevel nasal positive airway pressure ventilation in patients with heart failure. Respiration 1999; 66: 136-143 